\title{
Chemotherapy administration standards and guidelines: The development of a resource document
}

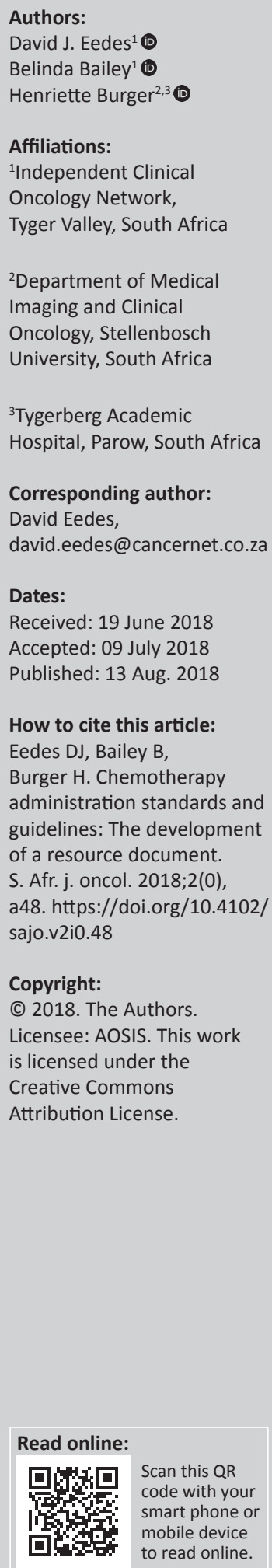

\begin{abstract}
There are no nationally recognised guidelines for the handling and administration of chemotherapy in South Africa. The Independent Clinical Oncology Network's Chemotherapy Administration Standards and Guidelines Resource Document was developed over 2 years and first introduced at a South African international oncology conference in 2017. A working group consisting of oncologists and oncology nurses was set up to address this deficiency. Pragmatic guidelines suitable to a wide range of local chemotherapy administration practices were developed using an iterative, multidisciplinary, collaborative process. The consensus was that these guidelines should be appropriate to the South African context. Safety, standard operational procedures, recommended professional competencies and training were central to the document. Guidelines for prescribing, storing, mixing, dispensing, administering and disposing of chemotherapy were included. Patient consent and involvement, patient and staff safety, recommended professional competencies, management of accidents and errors, error reporting and local legal requirements are dealt with in detail. The hope is that these guidelines will be used as a resource document for South African chemotherapy practices, both public and private. The document is supported by standard operating procedures and action steps. These were developed to promote the use of the guidelines and to support pragmatic quality assurance measures at practice level. These standards and guidelines will be regularly updated, based on needs identified and deficiencies noted.
\end{abstract}

\section{Introduction}

Cancer chemotherapy administration is a complex medical procedure. It requires high levels of competency and safety to prevent errors that may cause harm to patients and staff.

The modern era of oncology offers a wide range of treatment options for cancer patients. These novel chemotherapeutic and hormonal agents, targeted and immune therapies, and new combinations provide the opportunity to improve patient outcomes. ${ }^{1}$ To capitalise on these gains and optimise outcomes, safe, error-free and competent chemotherapy administration processes are crucial.

These guidelines recognise that:

- Oncology is a challenging field with high stakes and vulnerable patients.

- Chemotherapy has narrow margins of error (therapeutic index).

- Chemotherapy is toxic even at therapeutic doses.

- Chemotherapy regimens are often highly complex.

- Chemotherapy administration is a multidisciplinary process.

\section{Aims}

These guidelines are a resource document with high aims. They were developed to encourage and assist oncology professionals to adapt, develop and enhance their current chemotherapy administration procedures and processes. Despite the high standards set, the document takes a pragmatic approach that is adaptable to the varied resources and clinical situations of the South African oncology context. Improved patient care, patient outcomes and the safety and competency of chemotherapy staff are at its heart.

Central to its aims is the promotion of two concepts:

- error reduction

- safety. 
While the goals of chemotherapy are far more than just error reduction and safety, these are critical points of departure before other goals, such as improved treatment outcomes, improved patient experience and staff job satisfaction, can be considered.

\section{Error reduction in chemotherapy administration}

Chemotherapy administration errors are a recognised issue worldwide. Local statistics for chemotherapy administration errors are not known. Medical errors rank third amongst the most common causes of death in the United States. ${ }^{2}$ Walsh et al. ${ }^{3}$ observed an error rate of 8.2 per 1000 medication orders in adult cancer patients in the outpatient setting, of which $5 / 1000$ had the potential to cause harm and 1/1000 resulted in injury to the patient.

A 2013 study on chemotherapy found a rate of almost 17 errors per 1000 chemotherapy doses in a sample of handwritten orders. ${ }^{4}$ A study conducted in Turkey found that $83 \%$ of nurses reported one or more errors during chemotherapy preparation and administration. ${ }^{5}$ Studies have shown a wide range of errors including under- and overdosing, incorrect medicines used, as well as administration of chemotherapy to the wrong patients. ${ }^{6}$

To reduce errors, and any potential harm caused to patients receiving chemotherapy, several aspects were considered in the guideline development. These include recommendations on appropriate training and competency evaluation of all healthcare professionals involved in chemotherapy administration, adequate staffing and a clear chemotherapy administration process that follows the usual chemotherapy patient pathway, with practical checklists for use at critical points in this pathway. Standard operating procedures (SOPs) for each process were also developed.

In these guidelines emphasis is placed on the fact that competency is defined as a measure of the actual ability to perform specific duties and not purely a qualification or professional title. The SOPs and checklists are designed to be practical, comprehensive and useful in the promotion of attention to detail at every step of the chemotherapy administration pathway. ${ }^{7,8}$

\section{Safety}

Chemotherapy should be given in an environment where safety is prioritised. ${ }^{9}$

The guidelines consider the issue of safety in chemotherapy administration as being comprised of the following:

- the safety of patients receiving chemotherapy

- the safety of professional staff involved in the prescribing, storage, handling, preparation, dispensing, disposing and administering of chemotherapy.

These guidelines are an attempt to assist diverse practices in South Africa develop a culture of safety.

\section{Document development}

This document flows out of the quality assurance (QA) programme in chemotherapy administration of the Independent Clinical Oncology Network (ICON).

\section{Quality assurance in chemotherapy administration}

Independent Clinical Oncology Network introduced a QA programme in all their network chemotherapy facilities in 2012. This was an adaptation of the national core healthcare standards consisting of the domains developed by the Office of Health Standards Compliance that came out of the National Health Amendment Act of 2013. ${ }^{10}$ Further adaptions to make these domains both relevant and user friendly were made in 2015. ${ }^{11}$ This QA programme was modelled on the wellaccepted principles already in place for the high-quality QA programmes used in all the network radiotherapy units. ${ }^{12}$

The use of practical protocols, checklists and SOPs is known to reduce errors in medical practice. ${ }^{13,14}$ For the network chemotherapy administration QA programme, the focus was on a process-orientated approach. During the implementation and review of this process at practice level it became apparent that chemotherapy administration guidelines of varying standards and utility existed around the country. Professional competencies of the staff involved in this process were also not standardised.

Oncology nurses in the network identified the lack of local practical, standardised chemotherapy administration guidelines as problematic and expressed a need for such guidelines to assist them in this complex area of medicine.

Based on this feedback it was decided to develop a practical, process-orientated and patient-centric guideline resource document. The multidisciplinary nature of chemotherapy treatment of a cancer patient was recognised as a key element in the guidelines design.

\section{Standards and guidelines document development}

A working group was set up to drive the document development process. This group started by undertaking wider consultation and interaction with the various chemotherapy practices in the network to gauge the need for, and interest in, such guidelines. Once the need was confirmed the process of data collection started.

The working group's aim was to collate the best of various recognised local and international standards and guidelines for the administration of chemotherapy in a single document. This was done with the clear brief to ensure that the outcome would be a practical, user-friendly guide that was applicable and adaptable to the diverse requirements of the South African oncology environment.

The initial draft document developed was discussed in depth at a workshop at a national oncology conference. 
Following this, a larger working group, taking feedback from the workshop, debated the proposed content and layout of the guidelines. Based on these discussions and further interactions, a final draft document was drawn up. This draft was then circulated for input and comment and a final iteration was ratified. This document was then published on the ICON website for use, feedback and comment within the network. Standard operating procedures and checklists were then developed to support the main document and are available to the network on request.

The working group recognised that modern chemotherapy is complex and requires high levels of skill from various professions. For this reason, it was felt that the document should address the main professions involved in this process and that the multidisciplinary team approach needed to be highlighted. This dictated the design of the document.

\section{The chemotherapy administration multidisciplinary team}

Patients receiving chemotherapy are generally managed by a team that includes the oncologist, the oncology pharmacist and the oncology nurse. The working group felt that the use of standardised guidelines by each discipline involved in the chemotherapy process, along with good lines of communication between disciplines, has a greater potential to both reduce the risk of chemotherapy administration errors as well as enhance the experience for professionals and patients alike.

The guidelines document has had extensive input from oncologists and oncology nurses and so currently focusses on these two areas. The hope is that South African oncology pharmacists will contribute to the completion of this document by developing guidelines of their own.

The document also recognises that the chemotherapy patient has a central role in the process. For this reason, the document focuses on patient education in detail. Issues around informed consent, counselling and family inclusion in this process are highlighted.

The chemotherapy patient was used as the starting point from which the pathway through the whole chemotherapy process was developed. This pathway was based on the route taken by most patients who receive chemotherapy (Figure 1). The document was then developed following this stepwise process.

The multidisciplinary process is once again highlighted in this pathway. The working group felt it was important to promote the concept that chemotherapy administration should ideally involve the three trained professional groups from different, but complementary, disciplines standardly involved in chemotherapy administration. It was felt that this cross-disciplinary approach, along with the involvement of a well-educated patient, would have the best chance of ensuring the reduction of administration errors and optimising the patient's outcome.

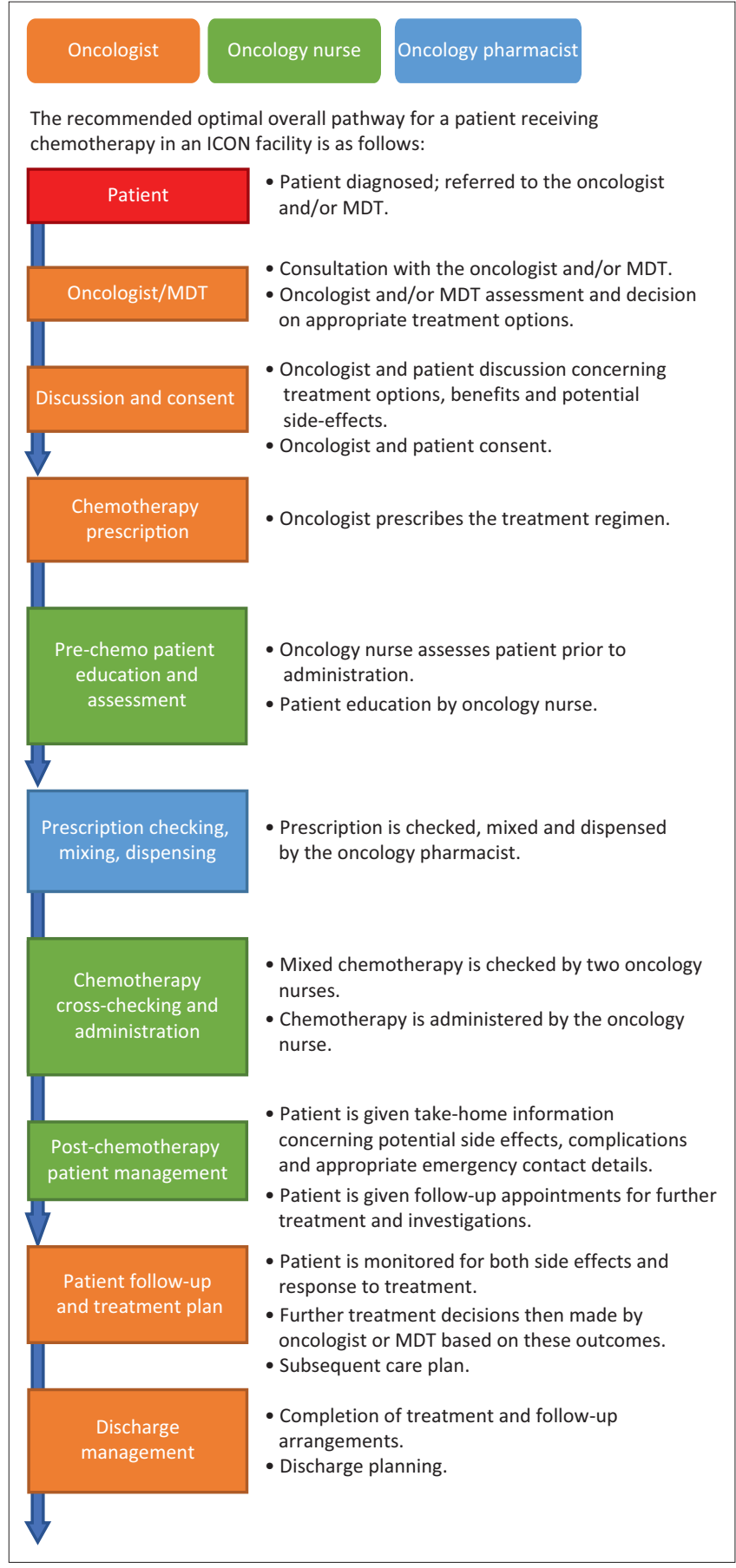

ICON, Independent Clinical Oncology Network; MDT, multidisciplinary team. FIGURE 1: The Chemotherapy Patient Pathway.

While the document describes processes and procedures that should be aimed for in the ideal chemotherapy setting, we are aware of the limitations and constraints that exist in our local environment. The hope is that, while all the steps and processes described may not be applicable to all chemotherapy practices in South Africa currently, the guidelines should be viewed as a starting point in the promotion of improved chemotherapy administration standards in South Africa.

The document was based on a patient-centric, multidisciplinary pathway (Figure 1). 


\section{Summary of the contents of the Chemotherapy Administration Standards and Guidelines resource document}

The document is divided into three sections:

1. General introduction

2. Role of the oncologist

3. Role of the oncology nurse.

A fourth section relating to the role of the oncology pharmacist would complete this document but, as of publication, we have not had input from the oncology pharmacist groups.

The 'General introduction' addresses the following topics:

- Aims and scope of the guidelines

- Errors in chemotherapy administration

- Current local guidelines for chemotherapy administration

- Control of medicines in South Africa

- The multidisciplinary nature of chemotherapy administration

- Competencies, skills and training

- Resources and medical cover

- Staff identification and patient confidentiality

- Expected basic knowledge.

The 'Role of the oncologist' section is further divided into the following headings and subheadings:

- The scope of practice for the oncologist

- General responsibilities

- Recommended competencies and skills

- The chemotherapy prescription/treatment plan

- General and legal principles

- Recommended chemotherapy prescription content

- Special considerations for oral chemotherapy prescription

- Informed consent and patient education

- General legal and ethical principles

- Recommended content of the informed consent process

- Oral chemotherapy - special considerations re consent

- Patient's role in consent and education - the 'vigilant partner'.

Other important areas covered under this section include record-keeping according to local legal principles, the management and reporting of errors in chemotherapy administration, chemotherapy adverse drug reactions and chemotherapy administration incidents.

The 'Role of the oncology nurse' section is structured to be of practical use in the clinical setting.

The introduction details the following:

- The complexity of chemotherapy treatment

- Risks of personal exposure to chemotherapy drugs

- The role of the chemotherapy patient and family.
'Competencies, skills and training' highlights the following points:

- Basic required skills such as venous cannulation and central access device care

- The oncology nurse's role in chemotherapy calculations.

The general duties of the oncology nurse are detailed:

- Receipt of chemotherapy

- Storage of chemotherapy

- Management of expired or damaged or contaminated drugs

- Preparation area (mixing area)

- Chemotherapy prescription

- Prescription standards

- Prescription check

- Verbal orders

- New orders

- Electronic systems

- Prescription double check.

'Chemotherapy mixing' looks at topics of importance to oncology nurses who mix chemotherapy in the clinic:

- Preparation

- Recommended personal protective equipment (PPE)

- Reconstitution

- Labelling of chemotherapy

- Issuing

- Transportation of chemotherapy

- Chemotherapy mixing register.

The central, practical section is titled 'Chemotherapy administration'. This starts by addressing the professional values of accountability and competence. It then describes the stepwise processes involved in the chemotherapy nurse's management of a cancer patient. To enhance the practical nature of this section it is divided into action steps (Box 1).

'Management of side-effects and adverse events' during chemotherapy administration looks at evidence-based approaches to the prevention and management of:

- Infusion reactions, hypersensitivity reactions and anaphylaxis

BOX 1: Action steps.

STEP 1.

Pre-chemotherapy patient assessment, education, and informed consent

1.1 Patient assessment prior to each chemotherapy administration

1.2 Patient education

1.3 Informed consent

STEP 2.

Administer pretreatment medication

STEP 3.

Chemotherapy administration steps

1.1 Patient identification

1.2 Double checking

1.3 Cannulation

1.4 Chemotherapy administration process

1.5 Completion of chemotherapy process

1.6 Documentation of chemotherapy process

STEP 4.

Post-chemotherapy patient management

STEP 5.

Oral chemotherapy

STEP 6.

Completion of chemotherapy administration records (documentation) 
- Extravasation

- Reporting of chemotherapy adverse drug reactions.

The section on 'Health and safety', a core element of these guidelines, focuses on both the safety of patients and the often unconsidered and unrecognised issue of chemotherapy staff health and safety:

A. Patient health and safety

- Facilities

- Staffing

- Equipment

- Emergency equipment

- Administration equipment

B. Worker health and safety

- Common routes of exposure

- Personal protective equipment

- Decontamination, cleaning and disinfection

- Worker contamination

- Chemotherapy spillage management.

Special attention is given to

- Staff medical surveillance:

- Routine surveillance

- Medical surveillance programmes

- Post-contamination surveillance.

The section 'Management and disposal of cytotoxic waste', an important safety and legal issue, covers the following subjects:

- Cytotoxic waste disposal

- Cytotoxic waste bins

- Sharp disposal containers

- Additional waste disposal recommendations:

- Contaminated disposable equipment

- PPE and cleaning equipment

- Contaminated non-disposable equipment

- Unused oral doses

- Patient waste and body fluids.

The oncology nurse guidelines conclude with the important topic of documentation.

Incident recording, reporting and management

- General principles of incident reporting and management

- Examples of incidents

- Key requirements for an incident report

- Documentation of an incident

- Implementation of QA improvements.

\section{Conclusion}

The ICON Chemotherapy Administration Standards and Guidelines is a single resource document that aims to fill an unmet need in local cancer care. ${ }^{15}$ The non-standardisation or paucity of chemotherapy administration standards, processes and competencies in South Africa are addressed in this document by using a patient-centric, multidisciplinary, practical approach.
The safety of both oncology patients receiving chemotherapy and staff involved in administering it served as a guiding principle.

The hope is that this resource document will support those chemotherapy professionals and practices in South Africa who expressed the desire to improve their current standards of chemotherapy administration.

The development of this document had the central aims of promoting improved safety and outcomes for oncology patients as well as improving the quality of the working environment for chemotherapy professional staff. Although the guidelines document aims for the highest of standards, it is designed to be adaptable to the varied settings in the South African medical environment. Implementation of the action steps in the form of appropriately adapted checklists on their own could be helpful in promoting a developmental improvement in chemotherapy administration safety even in resource-constrained environments.

The involvement of local oncology nurses in the development of this resource document must be recognised as significant. In South Africa, as in most parts of the world, oncology nurses play a central role in the complex process of chemotherapy administration, cancer patient care and ensuring that the highest standards are adhered to.

The guidelines document should be viewed as a work in progress with the aim to review and update it on a regular basis.

\section{Acknowledgements}

The authors would like to acknowledge the input of the core document development working group:

- Florence Arends, RN

- Andrea Brummer, BNursing (Wits)

- Lemohang Mosala, RN

- Shelley Muscat, RN.

We would also like to thank Prof. Raymond Abratt and Dr Anita Bonthuys for their support in writing this article.

\section{Competing interests}

The authors declare that they have no financial or personal relationships that may have inappropriately influenced them in writing this article.

\section{Authors' contributions}

D.E. drafted the first version of the manuscript. B.B. and H.B. contributed to the writing of the manuscript. D.E., B.B. and H.B. approved the final version.

\section{References}

1. Howlader N, Noone AM, Krapcho M, et al., editors. SEER Cancer Statistics Review, 1975-2014. Bethesda, MD: National Cancer Institute; 2017 [cited n.d.]. Based on November 2016 SEER data submission, posted to the SEER website [homepage on the Internet]. Available from: https://seer.cancer.gov/csr/1975_2014/ 
2. Makary MA, Daniel, M. Medical error-the third leading cause of death in the US BMJ. 2016:353:i2139. https://doi.org/10.1136/bmj.i2139

3. Walsh KE, Dodd KS, Seetharaman K, et al. Medication errors among adults and children with cancer in the outpatient setting. J Clin Oncol. 2009;27(6):891-896. https://doi.org/10.1200/JCO.2008.18.6072

4. Elsaid K, Truong T, Monckeberg M, et al. Impact of electronic chemotherapy order forms on prescribing errors at an urban medical center: Results from an interrupted time-series analysis. Int J Qual Health Care. 2013;25(6):656-663. https://doi.org/10.1093/intqhc/mzt067

5. Ulas A, Silay K, Akinci S, et al. Medication errors in chemotherapy preparation and administration: A survey conducted among oncology nurses in Turkey. Asian Pac J

6. Schulmeister L. Chemotherapy medication errors: Descriptions, severity, and contributing factors. Oncol Nurs Forum. 1999;26(6):1033-1042.

7. Chen C, Kan T, LiS, et al. Use and implementation of standard operating procedures and checklists in prehospital emergency medicine: A literature review. Am J Emerg Med. 2016;34(12):2432-2439. https://doi.org/10.1016/j.ajem.2016.09.057

8. Thomassen $\mathrm{O}$, Storesund A, Softeland E, et al. The effects of safety checklists in medicine: A systematic review. Acta Anaesthesiol Scand. 2014;58(1):5-18. https://doi.org/10.1111/aas.12207

9. Neuss MN, Gilmore TR, Belderson KM, et al. Updated American Society of Clinical Oncology/Oncology Nursing Society Chemotherapy Administration Safety 1262-1271 htts://doi.org/10.1200/JOP.2016.017905
10. National Health Amendment Act No 12 of 2013. Government Gazette Vol. 57724 July 201.3 [homepage on the Internet]. [cited n.d.]. Available from: https://www. gov.za/sites/www.gov.za/files/36702_gon529_0.pdf

11. Abratt RP, Eedes D, Bailey B, et al. Audits of oncology units - An effective and pragmatic approach SAMJ. 2017;107(6):493-496. https://doi.org/10.7196/ SAMJ.2017.v107i6.12356

12. Poortmans PM, Davis JB, Ataman F, et al. The quality assurance programme of the Radiotherapy Group of the European Organisation for Research and Treatment of Cancer: Past, present and future. Eur J Surg Oncol. 2005;31(6 SPEC. ISS.): 667-674.

13. Wrixon AD. New recommendations from the International Commission on Radiological Protection - A review. Phys Med Biol. 2008;53:R41-R60. https:// doi.org/10.1088/0031-9155/53/8/R01

14. Kouloulias VE, Poortmans PM, Bernier J, et al. The quality assurance programme of the Radiotherapy Group of the European Organization for Research and Treatment of Cancer (EORTC): A critical appraisal of 20 years of continuous efforts. Eur J Cancer. 2003;39:430-437. https://doi.org/10.1016/S0959-8049(02) 00113-2

15. Eedes D, Bailey B, editors. ICON Chemotherapy Administration Standards and Guidelines Resource Document - The role, responsibilities and requisite competencies of healthcare professionals involved in the administration of chemotherapy [homepage on the Internet]. 2017 [cited n.d. ]. Available from: https://iconsa.co.za/for-doctors/icon-accreditation/ 